\title{
Polymyositis masquerading as mitochondrial toxicity
}

\author{
K P Prime, S G Edwards, M R Pakianathan, J L Holton, F Scaravilli, R F Miller
}

Sex Transm Infect 2003;79:417-418

A 66 year old HIV infected male heavy smoker presented with arthralgia, myalgia, and weight loss which was originally ascribed to nucleoside induced mitochondrial toxicity. Despite withdrawal of antiretroviral therapy a proximal myopathy developed. Further investigation excluded malignancy. Polymyositis was diagnosed on muscle biopsy. The patient recovered completely with oral prednisolone. This case highlights the importance of muscle biopsy in HIV infected patients whose myopathy persists despite withdrawal of antiretroviral therapy and the need for thorough investigation of non-specific symptoms in HIV infected patients who are receiving antiretroviral therapy.

\footnotetext{
A
} 66 year old white male was found to be HIV-1 antibody positive in 1995. In December 1997 he began antiretroviral therapy with zidovudine, lamivudine, and didanosine. In the past he had been treated for primary syphilis in 1977. In September 1999 he had presumptive pulmonary tuberculosis, which was treated with quadruple antituberculous therapy for 6 months. There was no family history. He had smoked 20 cigarettes/day for 50 years, drank $<5$ units of alcohol/week and denied recreational drug use.

He presented in May 2001 with a 6 month history of generalised myalgia and arthralgia together with $5 \mathrm{~kg}$ weight loss. On examination, findings included widespread loss of subcutaneous fat and muscle bulk in the legs. Investigations showed a normal full blood count, urea and electrolytes, calcium and phosphate, glucose, $C$ reactive protein (CRP), thyroid function tests, PSA and serum angiotensin converting enzyme (ACE). An autoantibody screen, including rheumatoid factor, ANA, and ANCA was negative. Toxoplasma serology was negative. Syphilis serology showed no evidence of reactivation or re-infection. The CD4 count was $310 \times 10^{6} / 1$ and HIV viral load 4000 copies $/ \mathrm{ml}$. Abnormal results were obtained for venous lactate 3.95 (normal range 0.5-2.2) $\mathrm{mmol} / \mathrm{l}, \mathrm{LDH}=917$ (normal range 240-480) $\mathrm{U} / \mathrm{l}, \mathrm{CPK}=497$ (normal range 24-204) U/l, alkaline phosphatase $=195$ (normal range 45-122) $\mathrm{U} / \mathrm{l}$, and complement $\mathrm{C} 3=0.65$ (normal range 0.9-1.8) g/l. A chest radiograph showed an opacity adjacent to the left hilum. An abdominal ultrasound scan showed a normal sized liver with fatty infiltration and mild splenomegaly. Antiretroviral therapy was stopped as the presentation was thought to be due to nucleoside induced mitochondrial toxicity.

Over the next 5 months his symptoms became progressively worse with continuing malaise and night sweats, further muscle wasting in the legs, and difficulty standing up from sitting. Dysphagia for solids also developed. The CD4 count fell to $170 \times 10^{6} / 1$ and the viral load increased to 189200 copies $/ \mathrm{ml}$. At this time antiretroviral therapy was restarted with didanosine, efavirenz, and lopinavir.

Re-examination at this stage revealed a proximal myopathy $(\mathrm{MRC}=4 / 5)$. Muscle tenderness and fasciculation were absent. There was no associated rash or arthropathy; nail appearances were normal. Lactate, CPK, and alkaline phosphatase remained elevated. The haemoglobin had fallen to $8.1 \mathrm{~g} / \mathrm{dl}$ with a normal serum $\mathrm{Bl} 2$ and red cell folate. Serum iron, total iron binding capacity, and serum iron saturation were reduced. Parvovirus serology was negative. A 24 hour urinary protein and serum electrophoresis were normal; Bence Jones protein was not detected in urine. The chest radiograph remained abnormal. Fibreoptic bronchoscopy was normal and staining and culture of bronchoalveolar lavage fluid were negative for mycobacteria, bacteria, and fungi. Whole body PET scanning showed focal increased uptake of fluorodeoxyglucose by two nodular lesions in the left lower lobe of the lung, suggesting malignancy. A thoracic CT scan showed only non-specific nodular changes in both lower lobes. Upper gastrointestinal endoscopy was normal. Videofluoroscopy was normal but a barium swallow showed a cricopharyngeal prominence compatible with a myopathy. Electromyography showed a myopathy. Nerve conduction studies showed a mild axonal peripheral neuropathy with features of a myopathy. Muscle biopsy of vastus medialis, performed 3 weeks after restarting antiretroviral therapy, showed a predominantly endomysial inflammatory infiltrate composed of a mixture of $\mathrm{T}$ lymphocytes and macrophages with occasional B lymphocytes. No microvasculitis was seen and there was no evidence of an acute inflammatory or granulomatous process. The appearances were those of severe polymyositis (fig 1 ).

Oral prednisolone $60 \mathrm{mg}$ once daily was given for 1 month. This resulted in complete resolution of symptoms. This was mirrored by normalisation of the LDH, CPK, and alkaline phosphatase levels. A repeat PET scan on completion of prednisolone treatment and 3 months after the initial PET scan was normal. After 24 weeks on antiretroviral therapy the CD4 count had increased to $330 \times 10^{6} / 1$, the viral load had fallen to $<50$ copies $/ \mathrm{ml}$ and the patient's symptoms had resolved completely.

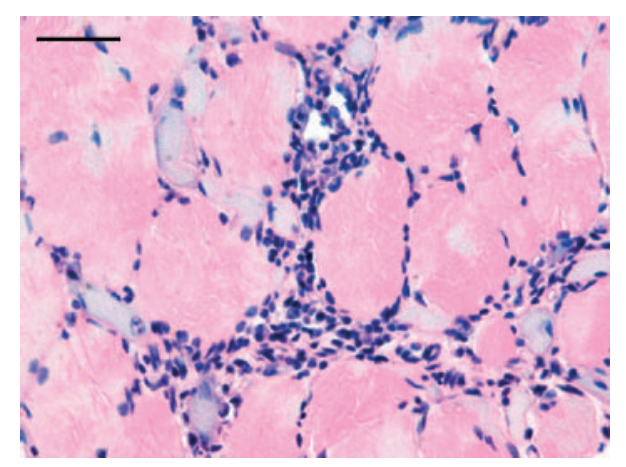

Figure 1 Haematoxylin and eosin preparation demonstrating increased variation in fibre size and endomysial lymphocytic infiltrate. Bar $=50 \mu \mathrm{m}$. 


\section{DISCUSSION}

In this patient nucleoside induced mitochondrial toxicity was suspected initially, given the history of widespread loss of subcutaneous fat and the loss of muscle bulk in the legs, together with hepatic steatosis and elevated CPK and lactate. Despite withdrawal of antiretroviral therapy symptoms progressed. Investigations excluded a paraneoplastic syndrome secondary to an underlying malignancy. Other causes of proximal weakness in HIV infected patients, including alcohol excess, hypo/hyperthyroidism, hypokalaemia, hypophosphataemia and collagen vascular disease, had been excluded before the patient underwent muscle biopsy. ${ }^{1}$ Without a muscle biopsy it may be difficult to discriminate between nucleoside induced myopathy and HIV associated polymyositis. Improvements following withdrawal of antiretroviral therapy support the former diagnosis. HIV associated polymyositis has been described in patients with myocardial and oesophageal involvement ${ }^{2}$ and also in the context of immune reconstitution; ${ }^{3}$ this latter scenario is unlikely, as the patient had been receiving antiretroviral therapy for several years at the time of his presentation.

This case demonstrates, firstly, that a myopathy with elevated CPK should alert the physician to a wide range of differential diagnoses. Chronic medical conditions should not be forgotten in an ageing cohort of HIV infected patients receiving antiretroviral therapy. ${ }^{4}$ Secondly, it highlights the importance of muscle biopsy to secure a diagnosis, if the proximal myopathy persists despite withdrawal of antiretroviral therapy.

\section{CONTRIBUTORS}

KPP wrote the first and co-wrote the final draft of the manuscript, and carried out a literature review; SGE and MRP were responsible for patient care and critically reviewed drafts of the manuscript; JLH and FS were responsible for interpretation of the muscle biopsy; RFM proposed the project, critically reviewed drafts of the manuscript and, together with KPP, wrote the final draft.

\section{Authors' affiliations}

K P Prime, S G Edwards, M R Pakianathan, Department of

Genitourinary Medicine, Camden Primary Care Trust, Mortimer Market Centre, London WCIE 6AU, UK

J L Holton, F Scaravilli, Department of Molecular Pathogenesis, Division of Neuropathology, Institute of Neurology, University College London, London WCIN 3BG, UK

R F Miller, Department of Sexually Transmitted Diseases, Royal Free and University College Medical School, University College London and Camden Primary Care Trust, Mortimer Market Centre, London WC1E 6AU

Source of income: nil.

Conflict of interest: none declared.

Correspondence to: Dr K P Prime, Department of Genitourinary Medicine, Camden Primary Care Trust, Mortimer Market Centre, London WCIE 6AU, UK; katiaprime@hotmail.com

Accepted for publication 12 May 2003

\section{REFERENCES}

1 Reveille JD. The changing spectrum of rheumatic disease in human immunodeficiency virus infection. Sem Arthritis Rheum 2000;30:147-66.

2 Fuster M, Negredo E, Cadafalch J, et al. HIV-associated polymyositis with lifethreatening myocardial and oesophageal involvement. Arch Intern Med 1999:159:1012.

3 Sellier P, Monsuez J-J, Evans J, et al. HIV-associated polymyositis during immune restoration with combination ARV therapy. Am J Med 2000;109:510-12.

4 AIDS/HIV quarterly surveillance tables 2002. Available at www.phls.co.uk.

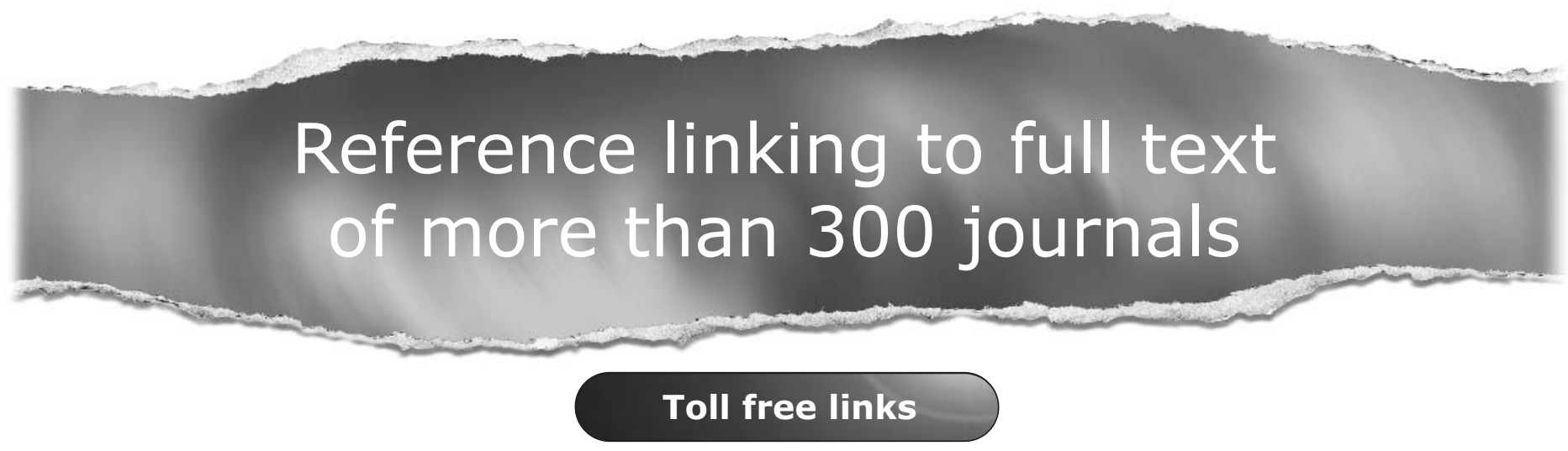

You can access the FULL TEXT of articles cited in Sexually Transmitted Infections online if the citation is one of the more than 340 journals hosted by HighWire (http://highwire.stanford.edu) without a subscription to that journal. There are also direct links from references tothe Medline abstract for other titles.

www.stijournal.com 